\title{
QUALIDADE DA BEBIDA EM ESPÉCIES E POPULAÇÕES DERIVADAS DE HÍBRIDOS INTERESPECÍFICOS DE COFFEA( $\left.{ }^{\natural}\right)$
}

\author{
ALCIDES CARVALHO (2,4), ALDIR ALVES TEIXEIRA (3), \\ LUIZ CARLOS FAZUOLI (24) O OLIVEIRO GUERREIRO FILHO (2)
}

\section{RESUMO}

Avaliou-se, em dois experimentos, a qualidade da bebida das especies Coffea canephora e $C$. congensis e derivados de hibridaçoes interespecfficas (C. canephora duplicado (dp) $\times$ C. arabica, C. canephora $\times$ C. eugenioides, C. arabica $\times$ C. denevrei dp e C. racemosa $\times$ C. arabical. Por tatar-se de análise de bebida de cates pouco conhecidos, avaliou-se a eficiencia de uma escala de 1 a 10 pontos em comparaçăo à escala de 0 a 5 pontos utilizada para C. arabica. Foram, também, acrescentadas pelos provadores indicaçōes relacionadas ao gosto da bebida. A escala de 6 pontos mostrou-se pouco eficaz na discriminaçăo dos tratamentos e a de 10 pontos, utilizada alternativamente, revelou-se mais eficiente nos dois experimentos. No primeiro, verificou-se, quanto à qualidade, uma superioridade dos grupos $C$. arabica $x$ C. denevrei $\mathrm{dp}$ e $C$. canephora $\mathrm{dp} \times C$. arabica pelas duas escalas. No segundo experimento, o grupo $C$. racemosa $\times C$. arabica apresentou a maior média, apesar de năo diferir, pela escala 1 , dos demais grupos. Pela escala 2 , superou, no entanto, os grupos $C$. canephora e $C$. congensis. Gostos incomuns foram observados nas amostras com relação a bebida. Atribuemse à grande diversidade do material analisado e à falta de familiarizaçăo dos provadores com espécies bem diferentes de $C$. arabica, certas discrepâncias com relaçăo a determinaçăo desses defeitos nas amostras analisadas.

Termoe de indexaçso: Coffes spp., hibridos interespec/ficos, qualidade de bebide.

\section{ABSTRACT \\ CUPPING QUALITY OF COFFEE SPECIES AND INTERSPECIFIC HYBRIDS}

Investigations on coffee cupping quality of the species $C$. canephora, C. congensis and several populations derived from interespecific crosses (C. canephora dp $\times$ C. arabica, $C$. canephora $\times C$. eugenioides, $C$.

(1) Resumos dos resultados reunidos neste trabatho foram apresentados na XXVII Reunião Anual da SBPC, realizede om Belo Horizonte, MG, de 9 a 16 de julho de 1975.

(2) Seção de Gonética, Instituto Agronómico (IAC). Caixa Postal 28, 13001 Campinas, SP.

(3) Instituto Biológico (IB), Caixa Postal 71 19, 01000, São Paulo, SP.

(4) Com bolsa de pesquise do CNPa. 
arabica $\times C$. dewevrei $\mathrm{dp}$ and $C$. racemosa $\times$ C. arabical were undertaken in two trials, in order to guide breeding programs using this germoplasm. Two grading systems were used for classification of the cupping quality, the normal six points scale used for $C$. arabica samples and a new one with 10 points, 1 for the lowest and 10 for the highest quality. Coffee liquorer experts graded the overall cupping quality and added remarks to any unpleasant taste noticed in the samples. The analysis of the data pointed out that both scales were not adequate for a complete characterization and discrimination of the individual samples. When coffee samples were grouped according to the species involved in the crosses, the results indicated the superiority of the $C$. arabica $x C$. denevrei dp and C. canephora dp $\times C$. arabica groups in the first trial. In the second tial the $C$. racemosa $\times C$. arabica group revealed the best average cupping quality, statistically superior to $C$. canephora and $C$. congensis. Most of the non-commercial coffees displayed unique tastes and were characterized in 13 unpleasant types. Due to the inherent diversity of the analysed material the liquorer experts had difficulty in classifying the several abnormal tastes influencing the classification of the mild standard sample. The results indicated the necessity of more accurate studies of the beverage produced by these species mainly when they participate in the breeding of Arabica coffee.

Index torme: Coffee spp., interspecific hybrids, coffee cupping quality.

\section{INTRODUÇÃO}

O banco de germoplasma de café do Instituto Aaronomico de Campinas conta com numerosos cultivares e variaçöes de Coffea arabica e de C. canephora, principais espécies economicas, além de um número razoável de espécies silvestres. Algumas das especies possuem alta capacidade produtiva, enquanto, em outras, a produçäo extremamente reduzida. Todas, no entanto, independentemente da capacidade produtiva, vem sendo estudadas com relaça à resistencia a pragas e moléstias, características valiosas para seu aproveitamento no melhoramento de $C$. erabica. Pouco se sabe, todavia, da qualidade da sua bebida. Sabe-se que todos os cultivares analisados de C. arabica, espécie que produz o melhor cafe e de maior cotaçăo no mercado internacional, dăo bebida de boa qualidade, desde que adequadamente preparados. O Robusta (C. cenephora), de bebida neutra, de qualidade inferior à de C. arabics, pode ser misturado a este, em determinadas proporç̋̄es, por nåo conferir gosto especffico e detrimental à bebida (GARRUTI et al., 1975). CARVALHO et al. (1990), estudando a bebida de $C$. dewevrei $\bar{c}$. Excelsa, que, apesar de produtivo, pouco conhecido, verificaram ser bem inferior à do Arábica. No entanto, sua adição de atr $23 \%$ ao Arábica de boa qualidade passaria despercebida aos provadores. Já o cafó Excelsa, em maiores proporçð̌es, conferiu um gosto bastante peculiar a bebida: desconhecido dos provadores, foi designado por "gosto estranho", acentuando-se com a maior participaçăo do Excelsa nas misturas. Escassas também sảo as informações sobre a bebida de outras especies de Coffea ou dos descendentes de suas hibridaçōes com C. arabica. 
Em vista do desconhecimento da qualidade da bebida de grande número de hibridos interespecfficos e de populaçöes descendentes desses híbridos existentes no banco de germoplasma, procurou-se avaliá-los visando orientar a seleção na escolha das melhores populações acerca desse aspecto.

\section{MATERIAL E MÉTODOS}

Dois experimentos foram estabelecidos para analisar a qualidade da bebida de diferentes espécies de Coffea e das populações derivadas de hibridações interespeclficas. Em ambos, o café foi colhido maduro, despolpado, fermentado, lavado, beneficiado e catado para retirar todos os grãos com defeito.

Analisaram-se seleçōes de cafeeiros pertencentes às espécies $C$. canephora e $C$. congensis, por serem rústicas e produtivas, $\theta$ os derivados das hibridações entre $C$. canephora duplicado (dp) e C. arabica, por serem populações básicas que deram origem ao café lcatu, de bastante interesse económico (MONACO et al., 1974). Estudaram-se os derivados de $C$. canephora x $C$. eugenioides, Dor essas espécies estarem provavelmente relacionadas com a origem de C. arabica (CARVALHO \& MONACO, 1967); os derivados de hibridações de $C$.arabica e $C$. dewevrei, esta última com número duplicado de cromossomos (dp), por apresentarem grande vigor e resistencia ao nematóide Meloidogyne exigua (FAZUOLI, 1981), e os derivados de C. racemosa x C. arabica, por terem originado populações em seleção para resistencia ao bicho-mineiro, Perileucoptera coffeella (MEDINA FILHO et al., 1977).

No primeiro experimento, analisaram-se 19 amostras, compreendendo quatro de $C$. congensis (coleçōes 2, 3, 5 e 6); uma de $C$. canephora (C1100-4); sete de $C$. arabica x C. dewevrei dp (CH 639-4, CH639-5, CH646-4, CH664-7, CH664-7-9, CH1365-7 e CH1852-9); quatro de C. canephora dp x C. arabica (LCH2460-1, LCH2460-2, $\mathrm{CH} 2460-5$ e $\mathrm{CH}_{2460-6)}$ e tês de $C$. canephora x C. eugenioides (CH3317-5-3, $\mathrm{CH} 6519-2$ e $\mathrm{CH} 6575-7)$. Na análise de grupos de tratamentos, realizada posteriormente, a amostra de $C$. canephora $\mathrm{C} 1100-4$ foi agrupada com as tes de $C$. canephora $\times C$. eugenioides, estabelecendo-se quatro grupos, a saber: grupo 1: C. congensis; 2: C. canephora dp $\times$ C. arabica; 3: C. canephora $\times$ C. eugenioides, e grupo 4: $C$. arabica $\times$ C. dewevei dp. Sem fazer parte da análise, usou-se uma amostra de $C$. arabica de boa qualidade como "tratamento de rotina" (TEIXEIRA, 1972). O experimento foi constitufdo de 19 tratamentos e 9 repetiçóes, em delineamento de blocos incompletos balanceados (COCHRAN \& COX, 1971). Por tratar-se de comparaçŏes entre cafés pouco conhecidos, adotou-se, tentativamente, nova escala de valores de 10 pontos (escala 2), comparando-a à de 0 a 5 pontos (escala 1), normalmente usada para C. arabica (TEIXEIRA, 1972). Na escala 2, 1 ponto corresponde à bebida Rio; 3 à Riada; 5 à Dura; 7 a Apenas Mole; 9 a Mole e 10 a Estritamente Mole. Na avaliação da qualidade, atravess da prova de Xícara, os provadores tentaram caracterizar o gosto diferente ou estranho percebido na bebida. A essas indicaçōes, consideradas defeitos, atribulram-se pontos negativos em uma escala crescente, relativa a sua intensidade, a saber: 
Gosto detectado

Pontos negativos

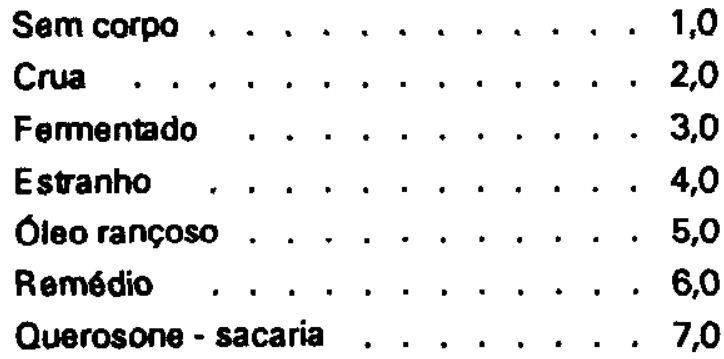

O número médio de pontos negativos dos tratamentos foi obtido pelo cálculo da média ponderada entre o número de pontos correspondentes aos defeitos e o número de amostras classificadas como tal.

A avaliaçăo das amostras foi feita por tres provadores, referindo-se os resultados ds médias das tres observaçöes.

No segundo experimento, analisaram-se as 22 amostras seguintes: tres de C. canephora (LC1277-3, Kouillou, CH1417-2; seis de C. congensis (Bangelan col. 9, Bangelan col. 11, Bangelan LC, Uganda col. 8, Uganda LC e CH1426-11): tês de C. canephore dp x C. arabica (CH2460-5, CH6171 e CH2834-6); seis de C. canephora $\times$ C. eugenioides (CH3317-1, LCH6575, CH6575-3, CH6575-4, CH6575-8 e LCH6575-11) e quatro de C. racemosa x C. arabica (C1195-5-6-1, C1195-5-6-2, C1195-5-8-3 e LC1 195-6). Acrescentaram-se ao experimento tos controles - um de bebida Dura, um de Riada o outro Apenas Mole. Para a análise, reuniram-se os tratamentos em grupos, a saber: 1: $C$. canephora; $2: C$. congensis; 3: $C$. canephora dp $\times$ C. arabica; 4: C. canephora $\times$ C. eugenioides, a 5: $C$. racemosa $\times$ C. arabica, ficando os padróes em um sexto grupo.

0 delineamento empregado foi o de látice balanceado $5 \times 5$ com seis repetições. Os tostes de bebida foram efetuados por tes provadores, utilizando-se a média das tres determinaçes para efeito de análise. Como no experimento anterior, avaliou-se a eficiencia de uma escala de 1 a 10 pontos (escala 2), em relação à de 0 a 5 pontos (escala 1).

Realizaram-se também, nesse experimento, observaçōes sobre o gosto da bebida, estando os defeitos e respectivos números de pontos negativos relacionados a seguir, em ordem crescente de prejulzo à qualidade da bebida:

Gos to detectado

Pontos negativos

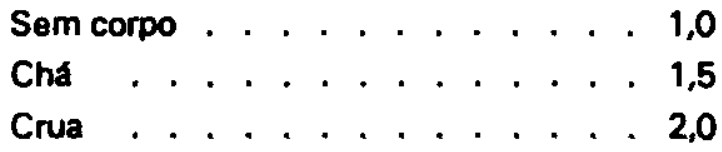




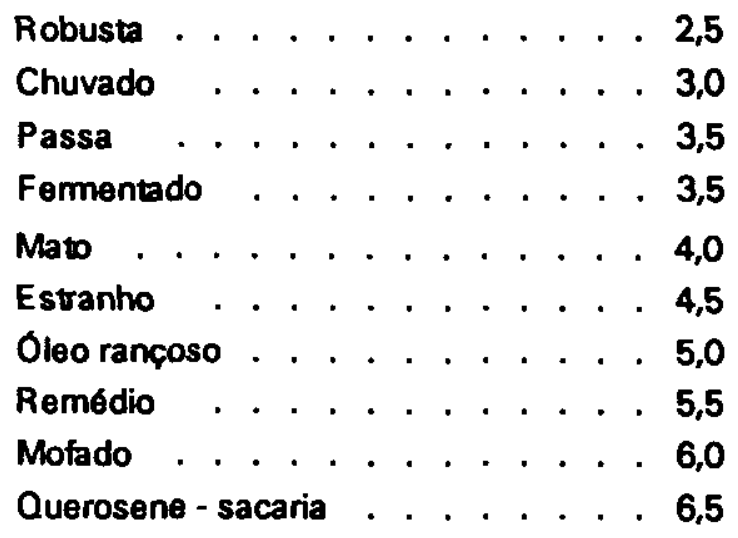

\section{RESULTADOS E DISCUSSÅO}

Observa-se, com relaçăo à escala 1 - Quadro 1 - que os derivados de C. arabica $\times$ C. dewevrei dp apresentaram múdias em geral superiores as das demais espécies. Pela análise de grupos, nota-se a superioridade estatrstica dos grupos C. arabica $\times$ C. dewevrei $\mathrm{dp}$ e C. canephora $\mathrm{dp} \times$ C. arabica, respectivamente, com médias de 3,70 e 3,42 pontos, em relaçăa ao $C$. canephora $\times C$. eugenioides $\theta$ C. congensis, com 2,95 e 2,88 pontos respectivamente. Pela escala 2 , o grupo $C$. arabica $\times$ C. dewevrei dp, com 7,64 pontos, igualou-se estatisticamente ao grupo C. canephora dp X C. arabica, com 7,34 pontos. Ambos, no entanto, diferiram significativamente do $C$. canephora $\times C$. eugenioides e do $C$. congensis, com, respectivaments, 6,44 6 6,17 pontos. $C$. dewewei apresenta uma bebida inferior ao Arábica, mas superior aos outros catás de seu grupo (Du Pasquier, apud DUBLIN, 1963). De acordo com CARVALHO et al. (1990), apesar da bebida ruim, C. dewevrei, quando misturado em ate $23 \%$ com o C. arabica de bebida boa, não provoca grandes alteraçoes na qualidade.

Verifica-se que alguns germoplasmas, como LCH2460-1, LCH2460-2 e $\mathrm{CH} 6575-7$, além da bebida de boa qualidade, năo apresentaram alteraçőes no seu gosto. Neste particular, cabo salientar que FAZUOLI et al. (1977), investigando a qualidade da bebida do cats Icatu - derivado de $\mathrm{CH} 2460$ (Arabusta) com sucessivos retrocruzamentos com C. arabica - verificaram também que ela pode ser considerada de boa qualidade, igualando-se mesmo do cultivar Acaía de C. arabica. Observaçöes de VAN DER VOSSEN (1985) indicaram, no entanto, tor - Arabusta bebida consideravelmente inferior a da seleçäo SL28 de C. arabica, nas condiçžes de Quenia. CAPOT (1972), analisando grande número de amostras do café Arabusta da Costa do Marfim, notou que a bebida 6 bem superior a do C. canephora traploide o semelhante a do Arábica, embora algumas amostras apresentassem bebida de qualidade intermediária entre $C$. arabica $C$. canephora. 
QUADRO 1. Média de pontos atriburdos à qualidade da bebida avaliada em escala de 0 a 5 pontos (escala 1) e de 1 a 10 pontos (escala 2) e números medios de pontos negativos relacionados ao gosto da bebida. Experimento 1

\begin{tabular}{|c|c|c|c|}
\hline \multirow{2}{*}{ Tratamentos } & \multicolumn{2}{|c|}{ Qualidade da bebida " } & \multirow{2}{*}{ Defeitos } \\
\hline & Escala 1 & Escala 2 & \\
\hline C. congensis & pontos & pontos & $\begin{array}{c}\text { pontos } \\
\text { negativos }\end{array}$ \\
\hline $\begin{array}{l}\text { col } 6 \\
\text { col } 3 \\
\text { col } 5 \\
\text { col } 2 \\
\text { Média }\end{array}$ & $\begin{array}{l}3,08 f-i \\
3,00 g-j \\
2,79 i j \\
2,63 j \\
2,88 b\end{array}$ & $\begin{array}{l}6,73 c-h \\
6,55 e-h \\
6,30 h i \\
5,08 j \\
6,17 b\end{array}$ & $\begin{array}{r}4,00 \\
3,56 \\
4,89 \\
11,67 \\
6,03\end{array}$ \\
\hline \multicolumn{4}{|c|}{ C. canephora dp x C. arabica } \\
\hline $\begin{array}{l}\text { LCH2460-1 } \\
\text { LCH2460-2 } \\
\text { CH2460-6 } \\
\text { CH2460-5 } \\
\text { Média }\end{array}$ & $\begin{array}{l}3,64 a-d \\
3,49 b-f \\
3,31 c-g \\
3,25 d-h \\
3,42 a\end{array}$ & $\begin{array}{l}7,55 a-d \\
7,39 a-f \\
7,25 a-g \\
7,16 a-h \\
7,34 a\end{array}$ & $\begin{array}{l}0,00 \\
0,00 \\
0,78 \\
0,44 \\
0,31\end{array}$ \\
\hline \multicolumn{4}{|c|}{ C. canephora $\times$ C. eugenioides } \\
\hline $\begin{array}{l}\mathrm{CH} 6575-7 \\
\mathrm{C} 1100-4 \\
\mathrm{CH} 6519-2 \\
\mathrm{CH} 3317-5-3 \\
\text { Média }\end{array}$ & $\begin{array}{l}3,16 \mathrm{e}-\mathrm{i} \\
2,97 \mathrm{~g}-\mathrm{j} \\
2,85 \mathrm{~h}-\mathrm{j} \\
2,80 \mathrm{ij} \\
2,95 \mathrm{~b}\end{array}$ & $\begin{array}{l}7,08 \mathrm{~b}-\mathrm{h} \\
6,51 \mathrm{gh} \\
6,67 \mathrm{~d}-\mathrm{h} \\
5,49 \mathrm{jj} \\
6,44 \mathrm{~b}\end{array}$ & $\begin{array}{r}0,00 \\
4,44 \\
2,56 \\
13,56 \\
5,14\end{array}$ \\
\hline \multicolumn{4}{|c|}{ C. arabica $\times$ C. dewevrei dp } \\
\hline $\begin{array}{l}\text { CH639-5 } \\
\text { CH639-4 } \\
\text { CH664-7 } \\
\text { CH664-7-9 } \\
\text { CH1365-7 } \\
\text { CH1852-9 } \\
\text { CH646-4 } \\
\text { Média }\end{array}$ & $\begin{array}{l}3,93 a \\
3,77 a b \\
3,77 a b \\
3,71 a-c \\
3,66 a-d \\
3,58 a-e \\
3,50 b-f \\
3,70 a\end{array}$ & $\begin{array}{l}7,97 a \\
7,82 a b \\
7,81 a b \\
7,61 a-c \\
7,59 a-c \\
7,42 a-e \\
7,25 a-g \\
7,64 a\end{array}$ & $\begin{array}{l}0,67 \\
1,00 \\
1,22 \\
1,33 \\
1,00 \\
2,11 \\
0,67 \\
1,14\end{array}$ \\
\hline $5 \%$ (Tratamentos) & 0,42 & 0,88 & - \\
\hline $5 \%$ (Grupos) & 0,34 & 0,51 & - \\
\hline CV $(\%)$ & 9,10 & 6,38 & - \\
\hline
\end{tabular}

* Médias de nove repetiçóes. Quando seguidas por letras iguais năo diferem estatisticamente entre si (Tukey $5 \%)$. 
Nas amostras derivadas das espécies $C$. congensis a C. canephora $x$ $x$ C. eugenioides, identificaram-se acentuados defeitos em relaçăo ao gosto e em maior freqüencia que nos outros derivados: apresentaram respectivamento, médias de 0,31 a 1,14 pontos negativos, enquanto os derivados de C. canephora $\times$ C. eugenioides e os de C. congensis apresentaram 5,14 e 6,03 pontos respectivamente - Quadro 1. Informaç̋es preliminares sobre a qualidade da bebida do hbrido entre $C$. cenephora e $C$. eugenioides foram apresentadas por LOUARN (1976), que verificou ter a bebida menos corpo que a de C. canephora, aproximando-se mais a de C. eugenioides.

Concluindo, a análise do primeiro experimento - Quadro 1 - revelou que a escala de 0 a 5 pontos foi pouco éficaz na discriminacăo de diferentes espécies de Coffea. Muitas amostras foram classificadas como de bebida Dura, embora apresentassem gostos distintos. O mesmo se verificou em relaço à escala de 10 pontos, também de pouca eficiencia.

No segundo experimento - Quadro 2 - os tratamentos năo diferiram estatisticamente entre si pela escala 1, nem mesmo do padrảo Apenas Mole, usado como controle. No entanto, todos foram significativamente superiores aos padröes de bebida Riada - Dura. A escala 2 foi um pouco mais eficiente que a 1, na discriminaçăo das médias dos tratamentos. Essa eficiencia foi, porém, limitada, como se verificou também no primeiro experimento. Da mesma forma, os provadores classificaram muitas amostras como de bebida Dura, embora caracterizada como de gosto diferento.

Para efeito de analise de orupos de tratamentos, os padrōes de bebida Riada, Dura e Apenas Mole foram reunidos em um mesmo grupo. No entanto, seria de pouco valor comparar a média desse grupo com as dos demais, de modo que foram apenas comparadas entre si. Derivados de C. racemose $\times$ C. arabica apresentaram, pela escala 1, a maior media de qualidade da bebida, 2,17 pontos, não diferindo estatisticamente, porém, dos demais grupos.

Pela escala de 1 a 10 pontos, o grupo de derivados de $C$. racemosa $\times C$. arabica, com 5,76 pontos, apresentou qualidade superior da bebida, não diferindo, no entanto, dos grupos de derivados de $C$. canephora dp $\times C$. arabica $\theta C$. canephora $\times C$. eugenioides, com 5,32 a 4,87 pontos respectivamente. Derivados de $C$. congensis, com 4,63 pontos, apresentaram a pior qualidade da bebida, inferior, estatisticamente, ao grupo C. recemose x C. arabica. Os resultados com os descendentes de C. recemosa $\times$ C. arabica, que deram bebida de qualidade superior, săo muito interessantes, pois numerosas populaçöes desse material vem sendo intensivamente estudadas com relaça a a resistencia ao bicho-mineiro, caracteristica proveniente de C. recemose. As amostras de C. canephore, de bebida semelhante de $C$. congensis, confirmam resultados de TEIXEIRA et al. (1979), que, analisando amostras de cafbs despolpado e em coco dessas espécies, coletadas em diferentes regibes cafeeiras, chegaram a mesma conclusão.

Com relaçăo a aheracós no gosto da bebida, os grupos, em geral, tiveram número do pontos semelhante. 
QUADRO 2. Média de pontos atribuídos à qualidade da bebida avaliada em escala de 0 a 5 pontos (escala 1) e de 1 a 10 pontos (escala 2) e números médios de pontos negalivos relacionados ao gos to da bebida. Experimento 2

\begin{tabular}{|c|c|c|c|}
\hline \multirow{2}{*}{ Tratamentos } & \multicolumn{2}{|c|}{ Qualidade da bebida } & \multirow{2}{*}{ Defieitos } \\
\hline & Escala 1 & Escala 2 & \\
\hline & pontos & pontos. & $\begin{array}{c}\text { pontos } \\
\text { negativos }\end{array}$ \\
\hline \multicolumn{4}{|l|}{ C. canephora } \\
\hline $\begin{array}{l}\text { LC1277-3 } \\
\text { Kouillou } \\
\text { CH1417-2 } \\
\text { M\&dia }\end{array}$ & $\begin{array}{l}2,05 a \\
2,00 a \\
1,92 a \\
1,99 a b\end{array}$ & $\begin{array}{l}4,83 b c \\
4,78 b c \\
4,39 b c d \\
4,67 b c\end{array}$ & $\begin{array}{l}7,42 \\
7,67 \\
8,67 \\
7,92\end{array}$ \\
\hline \multicolumn{4}{|l|}{ C. congensis } \\
\hline $\begin{array}{l}\text { Bangelan col. } 9 \\
\text { Uganda col. } 8 \\
\text { Bangelan LC- } \\
\text { Uganda LC } \\
\text { CH1426-11 } \\
\text { Bangelan col. } 11 \\
\text { Média }\end{array}$ & $\begin{array}{l}2,05 a \\
2,00 a \\
1,95 a \\
2,00 a \\
1,95 a \\
1,92 a \\
1,98 a b\end{array}$ & $\begin{array}{l}4,89 b c \\
4,78 b c \\
4,72 b c \\
4,56 b c d \\
4,50 b c d \\
4,34 c d \\
4,63 b c\end{array}$ & $\begin{array}{l}5,00 \\
8,08 \\
4,33 \\
6,33 \\
7,25 \\
8,00 \\
6,50\end{array}$ \\
\hline \multicolumn{4}{|c|}{ C. canephora $\mathrm{dp} \times$ C. arabica } \\
\hline $\begin{array}{l}\mathrm{CH} 2460-5 \\
\mathrm{CH} 6171 \\
\mathrm{CH} 2834-6 \\
\text { Média }\end{array}$ & $\begin{array}{l}2,20 a \\
2,12 a \\
2,05 a \\
2,12 a\end{array}$ & $\begin{array}{l}5,39 a b c \\
5,39 a b c \\
5,17 a b c \\
5,32 a b\end{array}$ & $\begin{array}{l}5,42 \\
6,58 \\
4,00 \\
5,33\end{array}$ \\
\hline \multicolumn{4}{|c|}{ C. canephora $\times$ C. eugenioides } \\
\hline $\begin{array}{l}\text { CH3317-1 } \\
\text { LCH6575 } \\
\text { LCH6575-11 } \\
\text { CH6575-3 } \\
\text { CH6575-8 } \\
\text { CH6575-4 } \\
\text { Média }\end{array}$ & $\begin{array}{l}2,20 \mathrm{a} \\
2,05 \mathrm{a} \\
1,97 \mathrm{a} \\
2,05 \mathrm{a} \\
1,95 \mathrm{a} \\
1,92 \mathrm{a} \\
2,02 \mathrm{ab}\end{array}$ & $\begin{array}{l}5,61 a b c \\
4,83 b c \\
4,78 b c \\
4,72 b c \\
4,67 b c \\
4,61 b c d \\
4,87 a b c\end{array}$ & $\begin{array}{l}5,33 \\
6,25 \\
6,00 \\
6,75 \\
4,50 \\
6,17 \\
5,83\end{array}$ \\
\hline \multicolumn{4}{|c|}{ C. racemosa $\times$ C. arabica } \\
\hline $\begin{array}{l}\text { C1195-5-8-3 } \\
\text { LC1195-6 } \\
\text { C1195-5-6-2 } \\
\text { C1195-5-6-1 } \\
\text { Média }\end{array}$ & $\begin{array}{l}2,42 a \\
2,07 a \\
2,10 a \\
2,10 a \\
2,17 a\end{array}$ & $\begin{array}{l}6,39 a \\
5,72 a b \\
5,55 a b c \\
5,39 a b c \\
5,76 a\end{array}$ & $\begin{array}{l}2,75 \\
5,08 \\
4,50 \\
4,42 \\
4,19\end{array}$ \\
\hline \multicolumn{4}{|l|}{ Padróes de bebida } \\
\hline $\begin{array}{l}\text { Riada } \\
\text { Dura } \\
\text { Apenas Mole } \\
\text { Média } \\
5 \% \text { (Tratamentos) } \\
5 \% \text { (Grupos) } \\
\text { CV }(\%)\end{array}$ & $\begin{array}{c}1,13 b \\
1,22 b \\
2,48 a \\
1,61 b \\
0,58 \\
0,45 \\
13,70\end{array}$ & $\begin{array}{c}3,28 \mathrm{de} \\
2,95 \mathrm{e} \\
6,28 \mathrm{a} \\
4,17 \mathrm{c} \\
1,33 \\
0,62 \\
12,75\end{array}$ & $\begin{array}{l}3,75 \\
2,17 \\
4,67 \\
3,53 \\
- \\
- \\
-\end{array}$ \\
\hline
\end{tabular}

\footnotetext{
- Médias de seis repetiçסes. Quando seguidas de letras iguais náo diferem estatisticamente entre si (Tukey $5 \%)$.
} 
Os provadores de café são especializados em classificar a bebida em uma escala de 0 a 5 pontos, respectivamente, Rio, Riada, Dura, Apenas Mole, Mole e Estritamente Mole. Esses padrōes se referem, no entanto, a bebida do café Arábica. Devido à grande diversidade do material analisado e ao fato de não estarem familiarizados com essa variação, os provadores tiveram dificuldade em identificar alguns dos defeitos, os quais influenciaram, provavelmente, sua classificação (Quadro 2). Isso talvez explique a razão de o padrão de bebida Dura ter sido classificado com número menor de pontos que o da Riada $\theta$, também, de 0 padrão de bebida Apenas Mole ter apresentado defeitos na bebida, de natureza semelhante a encontrada nas amostras das espécies $\theta$ dos hibridos analisados.

Os resultados desses dois experimentos ilustram bem a dificuldade de determinar a qualidade da bebida de especies silvestres e seus derivados. Há necessidade de ampliar as investigaçoes a esse respeito, principalmente com relação aos germoplasmas mais utilizados em programas prioritários de melhoramento de $C$. arabica, visando ao aproveitamento dessas especies como fonte de resistência a pragas e moléstias.

\section{AGRADECIMENTOS}

Os autores agradecem a colaboração aos provadores José Luiz Barbosa de Toledo, Moacyr Aprígio de Menezes e José Canabrava Barbosa, do Instituto Brasileiro do Café, pelas provas realizadas, e ao técnico Antonio Chaves de Carvalho, que efetuou a torração de todas as amostras.

\section{REFERENCIAS BIBLIOGRÁFICẠS}

CAPOT, J. L'amélioration du caféier em Côte d'ivoire: les hybrides "Arabusta". Cafe Cacao The, Paris, 16(1):3-18, 1972.

CARVALHO, A.; FAZUOLI, L.C.; TEIXEIRA, A.A. \& GUERREIRO FILHO, 0. Aproveitamento do café Excelsa (Coffea dewevren) em misturas com o caf́ Arábica (C. arabica). Bragantia, Campinas, 49(2):335-343, 1990.

\& MONACO, L.C. Genetic relationships of selected Coffes species. Ciencia e Cultura, Sảo Paulo, 19(1):151-165, 1967.

COCHRAN, W.G. \& COX, G.M. Diseños experimentales. Chapingo, Trillas, 1971. $653 p$.

DUBLIN, P. Le caféier Excelsa en République Centrafricaine: Etude de la graine. Café Cacao The, Paris, 7(1):6-21, 1963.

FAZUOLI, L.C. Resistance of coffee to the root-knot nematode species Meloidogyne exigua and $M$. incognita. In: COLLOOUE INTERNATIONAL SUR LA PROTECTION DES CULTURES TROPICALES. Lyon, 1981. Résumés. Lyon, Fondation Scientifique de Lyon et du Sud-Est, 1981. p.57.

CARVALHO, A.; MONACO, L.C. \& TEIXEIRA, A.A. Qualidade da bebida do café Icatu. Bragantia, Campinas, 36:165-172, 1977. 
GARRUTI, R.S.; CARVALHO, A. \& TOSELLO, Y. Qualidade da bobida em blends de cafés Arábica e Robusta. In: CONGRESSO BRASILEIRO DE PESQUISAS CAFEEIRAS, 3., Curitiba, 1975. Resumos. Rio de Janeiro, IBC-GERCA, 1975. p.33.

LOUARN, J. Hybrides interespécifiques entre Coffea canephore Pierre et $C$. eugenioides Moore. Cafe Cacao The, Paris, 20(1):33-52, 1976.

MEDINA FILHO, H.P.; CARVALHO, A. \& MEDINA, D.M. Germoplasma de Coffea racemosa e seu potencial no melhoramento do caffeeiro. Bragantia, Campinas, 36:XLIIIXIVI, 1977. (Nota, 11)

MONACO, L.C.; CARVALHO, A. \& FAZUOLI, L.C. Melhoramento do cafeoiro: germoplasma de cafe lcatu e seu potencial no melhoramento. In: CONGRESSO BRASILEIRO SOBRE PESOUISAS. CAFEEIRAS, 2., Poços de Caldas, 1974. Resumos. Rio de Janeiro, IBC-GERCA, 1974. p.103.

TEIXEIRA, A.A. A tecnica experimental do degustaçăo do café. Piracicaba, ESALQ, 1972. 99p. Tese (Doutoramento).

; CARVALHO, A. \& FAZUOLI, L.C. Avaliação da bebida o outras caracteristicas de cultivares de Coffes canephora a Coffea congensis. Bragantis, Campinas, 38:37-46, 1979.

VAN DER VOSSEN, H.A.M. Coffee selection and breeding. In: CLIFFORD, M.N. \& WLLSON, K.C., eds. Coffee: botany, biochemistry.and production of beans and beverage. Westport, AVI Publishing, 1984. p.48-96. 